Check for updates

Cite this: RSC Adv., 2019, 9, 24175

\title{
Dispersion of microcapsules for the improved thermochromic performance of smart coatings $\dagger$
}

\begin{abstract}
Sireesha Pedaballi, Chia-Chen Li ${ }^{*}$ * and Ya-Jun Song
Microcapsules are widely applied in coatings; however, there are very few reports on the dispersion of microcapsules in the coating material or the effects of dispersion on their performance. Herein, the efficiency of three types of dispersants bearing distinctive functional groups for the dispersion of concentrated thermochromic microcapsules in a solvent and coating resin is studied. The dispersion properties of $35 \mathrm{wt} \%$ thermochromic microcapsules in toluene and in coating resin are investigated by measuring their sedimentation and rheological behavior. Interactions between the dispersant and microcapsules are characterized by Fourier transform infrared spectroscopy in order to identify the dominant dispersion mechanism. The physical quality and thermochromic performance of the coating films are characterized by electron microscopy observations and temperature variation tests. The most effective dispersant for thermochromic microcapsules is determined. Compared to the coating film containing non-dispersed microcapsules, the film with well-dispersed microcapsules shows improved surface flatness, with few or no pores in the microstructure. In addition, the color of the film with welldispersed thermochromic microcapsules shows faster response to temperature variation, resulting in complete and uniform color transformation.
\end{abstract}

Received 24th June 2019
Accepted 29th July 2019

DOI: $10.1039 / c 9 r a 04740 a$

rsc.li/rsc-advances

compatible with the thickness of the coating layer. That is,

\section{Introduction}

Research on the use of microcapsules for advanced and smart materials has been pursued since $1993 ;^{1-3}$ this is because their hollow structure allows the loading of a variety of core materials, leading to potential applications such as autonomous restoration materials, ${ }^{1-7}$ anti-corrosion coatings, ${ }^{7-11}$ energy storage materials, ${ }^{12,13}$ and pharmaceutical and biomedical products. ${ }^{14-16}$ In addition, microcapsules are the key component for a variety of functional coatings. For example, embedding photochromic or thermochromic microcapsules endows the coating with a color-change function that can be triggered by UV irradiation or temperature variation, respectively. Functional coatings have many applications and a large market in industrial products such as plastics, fabrics, printing, paints, and inks. So far, the best process for the synthesis of microcapsules is to encapsulate the functional agent inside a thin shell via in situ polymerization. ${ }^{2,4-13}$ The most commonly reported shell materials are poly(urea formaldehyde) (PUF),,$^{2,5,6,10-13}$ polyurethane (PU), ${ }^{7,17-19}$ or composites of the two, and the functional agents in the core can be of either in the liquid or solid phase.

For coating applications, an important consideration is that the embedded microcapsules should have a size that is

Institute of Materials Science and Engineering, Department of Materials \& Mineral Resources Engineering, National Taipei University of Technology, Taipei 10608, Taiwan.E-mail: ccli@mail.ntut.edu.tw

$\dagger$ Electronic supplementary information (ESI) available. See DOI: 10.1039/c9ra04740a a thick coating may allow the embedding of either large or small microcapsules, while a thin coating is only suitable for small microcapsules; this is in order to avoid creating a rough coating surface of poor physical quality. At the same time, microcapsules with a size less than $10 \mu \mathrm{m}$ generally agglomerate more easily, which also is a detriment to coating quality and limits their applications. Although the idea of using microcapsules in coatings has been proposed for more than two decades, no discussion about the dispersion effects of embedded microcapsules on coating quality has been reported in the literature. In this investigation, the dispersion properties of a poly(melamine formaldehyde) (PMF)-based thermochromic microcapsule in a commonly used polymer base, a UV-curable polyurethane acrylate (golden oil), ${ }^{20}$ are studied. A phosphatebased dispersant that can efficiently improve the dispersion and distribution uniformity of microcapsules in golden oil through a simple mixing way is revealed. This simple mixing is more a physical method that can make the dispersion of microcapsules easier to handle than other chemical methods with using silanes or expensive reagents and through complicated reactions. ${ }^{21-23}$ In addition, this study also propose a possible dominant dispersion mechanism of the added dispersants for the PMF microcapsules. The dispersion properties were determined using rheological measurements and sedimentation experiments, and the effects of microcapsule dispersion on the physical microstructure and the thermochromic performance of the coatings were clarified. 


\section{Results and discussion}

\section{Agglomeration of microcapsules}

Fig. 1(a) shows the morphology of the as-received thermochromic microcapsules, and the shell material of which is poly(melamine formaldehyde) (PMF). The microcapsules have a round shape with a size range $2-10 \mu \mathrm{m}$, and it can be seen that the microcapsules exhibit a serious agglomeration problem. The agglomerated size is larger than $20 \mu \mathrm{m}$, which makes it difficult to use in applications that require the embedding of microcapsules in a thin coating. On the other hand, it can be seen that there are some impurities and small particles distributed on the microcapsule surface, giving the microcapsules a high surface roughness. The small particles could be small microcapsules and the impurity could be residue from the incomplete polymerization of PMF, additives for polymerization, or an excess of the core material. To determine the compatibility and dispersibility of microcapsules in an organic medium, $35 \mathrm{wt} \%$ (relative to the weight of solvent) of microcapsules were suspended in toluene and ultrasonicated at high power. Notably, the high shear force provided by high-power ultrasonication is generally useful in de-agglomerating particles, especially effective for soft agglomerates, ${ }^{\mathbf{6}, 24}$ but unfortunately the microcapsules remained agglomerated after ultrasonication; in fact, the agglomeration was worse than the original state with a size of 50-100 $\mu \mathrm{m}$, as shown in Fig. 1(b). In addition, it was found that the microcapsule surface became clearer and smoother after contact with toluene, suggesting that the impurity on the microcapsule surface could be dissolved in

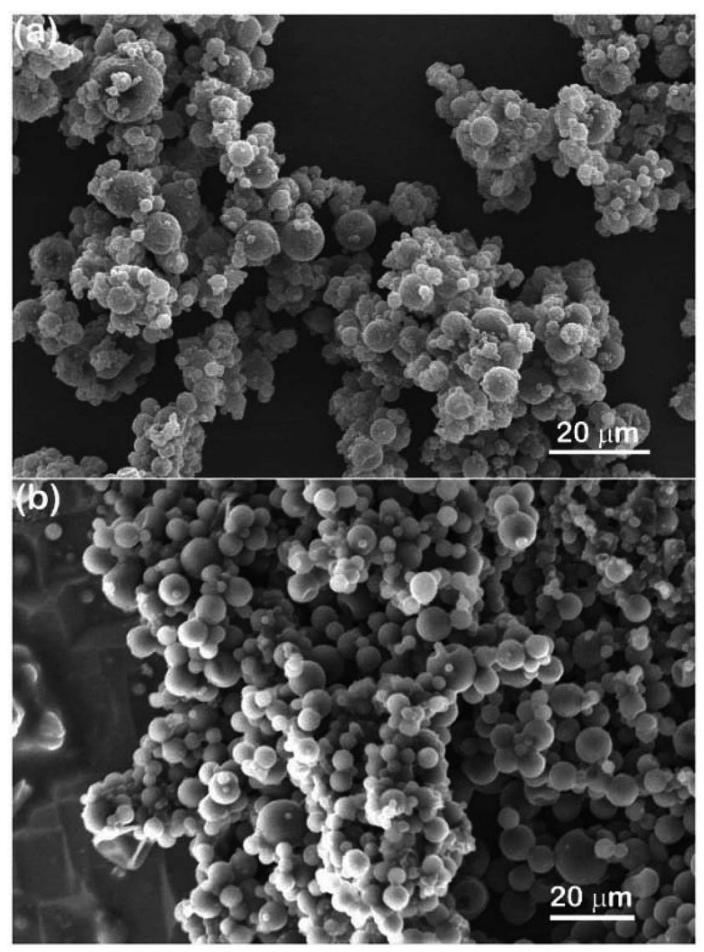

Fig. 1 SEM images showing structural morphology of as-received thermochromic microcapsules (a) before and (b) after high-power ultrasonication in toluene. toluene, which corresponds well to the above-mentioned possible identities of the impurity; however, the reduction in the surface roughness did not help to de-agglomerate the microcapsules.

\section{Dispersion in organic solvent}

In order to successfully apply the microcapsules in organic coatings, three dispersants, $\mathrm{OA}, \mathrm{OP}_{3}$, and $\mathrm{OP}$, were used to disperse the thermochromic microcapsules. The chemical structures of these three dispersants are shown in Fig. 2. They all contain oleophilic hydrocarbon chains and are miscible with organic media such as toluene and golden oil (GO). Fig. 3 compares the dispersion stability of $35 \mathrm{wt} \%$ thermochromic microcapsules in toluene with the addition of $0-20 \mathrm{wt} \%$ (relative to the weight of the microcapsules) of the different dispersants; sedimentation was recorded at various time periods after magnetic stirring for $10 \mathrm{~min}$. Fig. 3( $\left.\mathrm{a}_{0}\right)-\left(\mathrm{c}_{0}\right)$ shows the initial state of the suspensions with the addition of $\mathrm{OA}, \mathrm{OP}_{3}$, and $\mathrm{OP}$, respectively; for each case, the samples from left to right represent added concentrations of $0,5,10,15$, and $20 \mathrm{wt} \%$. After $30 \mathrm{~min}$ of sedimentation, the suspensions with OA and $\mathrm{OP}_{3}$ addition began to show the settling of microcapsules in Fig. $3\left(\mathrm{a}_{05}\right)$ and $3\left(\mathrm{~b}_{05}\right)$, respectively, while the suspensions with $5-20 \mathrm{wt} \%$ OP addition remained well dispersed in Fig. $3\left(\mathrm{c}_{05}\right)$. After $24 \mathrm{~h}$ of sedimentation, Fig. $3\left(\mathrm{a}_{24}\right)-\left(\mathrm{c}_{24}\right)$ shows that only the suspensions with OP addition exhibit good dispersion stability at concentrations of 5-20 wt\% (Fig. 3( $\left.\mathrm{c}_{24}\right)$ ). For the suspensions with OA (Fig. $3\left(\mathrm{a}_{24}\right)$ ) and $\mathrm{OP}_{3}$ (Fig. $3\left(\mathrm{~b}_{24}\right)$ ), almost complete sedimentation was obtained, except for the cases of $20 \mathrm{wt} \%$ OA and 15 and $20 \mathrm{wt} \% \mathrm{OP}_{3}$. After sedimentation for $48 \mathrm{~h}$, the top
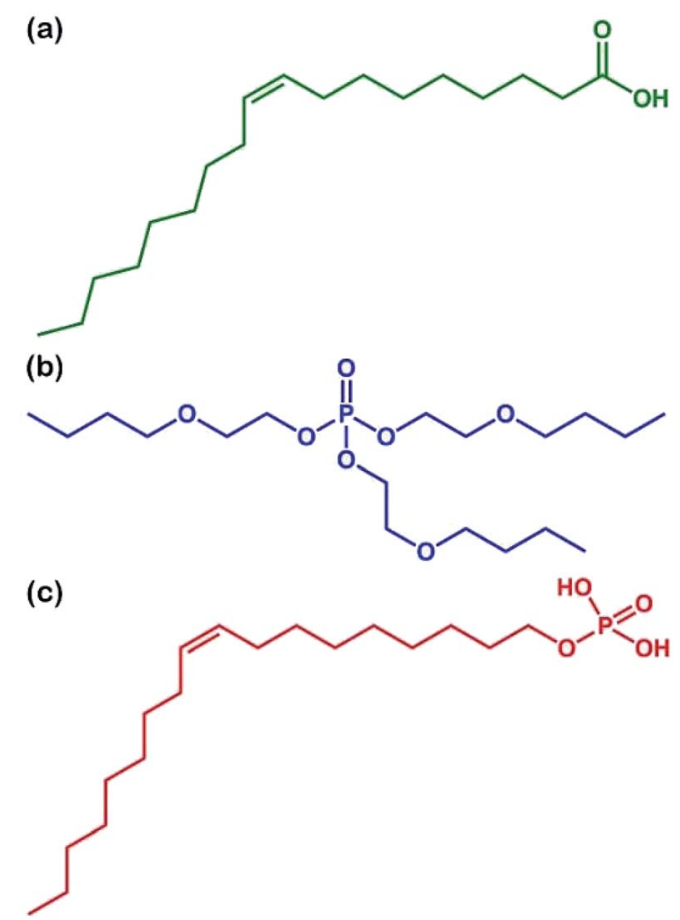

Fig. 2 Chemical structures of (a) $\mathrm{OA}$, (b) $\mathrm{OP}_{3}$, and (c) OP. 

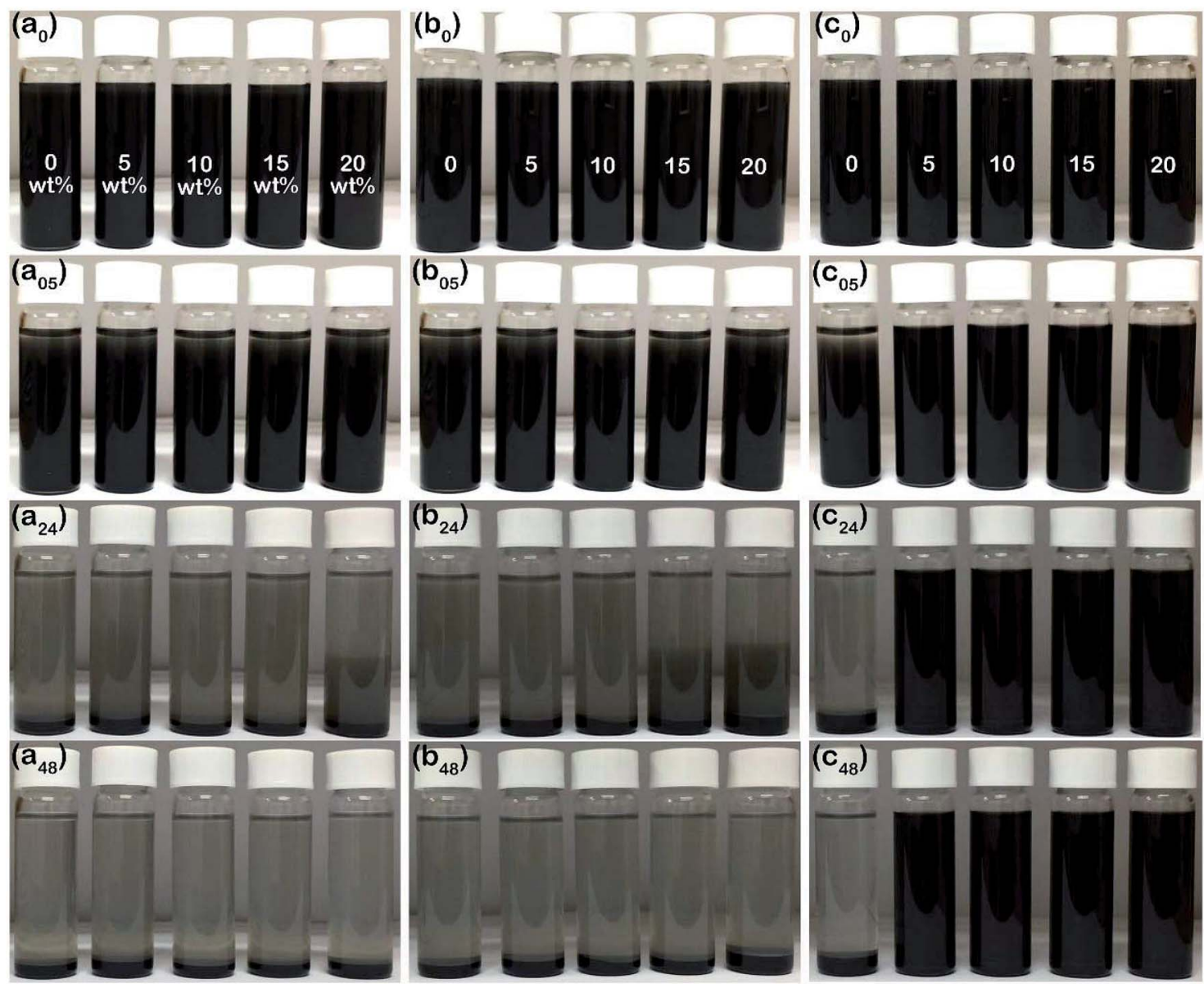

Fig. 3 Sedimentation of toluene suspensions containing 35 wt\% microcapsules with the addition of $\left(a_{0}-a_{48}\right) O A,\left(b_{0}-b_{48}\right) O P_{3}$, and $\left(c_{0}-c_{48}\right) O P$ for $0 \mathrm{~min}, 30 \mathrm{~min}, 24 \mathrm{~h}$, and $48 \mathrm{~h}$; the samples from left to right in each photo represent increasing concentration of dispersants of 0, 5, 10, 15, and 20 wt\%.

layer of the suspensions became clearer for $5-20 \mathrm{wt} \%$ OA (Fig. $3\left(\mathrm{a}_{48}\right)$ ) and $\mathrm{OP}_{3}$ (Fig. $3\left(\mathrm{~b}_{48}\right)$ ). The sedimentation behavior of the samples with $\mathrm{OA}$ and $\mathrm{OP}_{3}$ being similar to that of the sample without dispersant addition indicates that dispersion with these two dispersants is less efficient. Interestingly, the suspensions with OP (Fig. 3( $\left.\mathrm{c}_{48}\right)$ ) remained stably dispersed, even at a low concentration of $5 \mathrm{wt} \%$, showing the excellent dispersion efficiency of OP. Furthermore, the dispersion of microcapsules in the suspensions was observed by SEM. Fig. 4 compares microcapsules dried from suspensions containing 5 wt $\%$ of OA (Fig. 4(a)), $\mathrm{OP}_{3}$ (Fig. 4(b)), and OP (Fig. 4(c)). It can be seen that the microcapsules are significantly agglomerated in the former two cases using $\mathrm{OA}$ and $\mathrm{OP}_{3}$ as the dispersant, while agglomeration is insignificant when the dispersant is OP.

\section{Dispersion in golden oil}

For thermochromic applications, the microcapsules are typically incorporated into resin-related coating materials. The dispersion of thermochromic microcapsules in a commonly used GO with and without the addition of different dispersants was characterized by rheology measurement. Fig. 5(a)-(c) shows rheological curves of various GO mixtures containing $35 \mathrm{wt} \%$ microcapsules and 0-20 wt\% of different dispersants. Because GO and dispersants have their own rheological properties which would contribute to the rheological behavior of the mixtures, the rheology shown in Fig. 5 is the relative viscosity $\left(\eta_{\mathrm{r}}\right)$ rather than the apparent viscosity $(\eta)$ as a function of shear rate, in order to avoid misidentification for the dispersion of microcapsules. The value of $\eta_{\mathrm{r}}$ was obtained by dividing the measured $\eta$ by the viscosity $\left(\eta_{0}\right)$ of the continuous phase comprised of only GO and the dispersant at various concentrations, as represented by eqn (1): $:^{24-26}$

$$
\eta_{\mathrm{r}}=\eta / \eta_{0}
$$

Using eqn (1), the rheological contributions of GO and the dispersants can be excluded from $\eta_{\mathrm{r}}$. In theory, a lower value of $\eta$ means better flowability of a mixture, but a lower $\eta_{\mathrm{r}}$ represents improved dispersion. In the case of $0-20 \mathrm{wt} \%$ OA in Fig. $5(\mathrm{a})$, the mixture without $\mathrm{OA}$ exhibits an obvious shear-thinning behavior, i.e., the value of $\eta_{\mathrm{r}}$ decreases with an increase of shear rate, indicating the presence of agglomerates. ${ }^{24-26}$ The decreasing value of $\eta_{\mathrm{r}}$ with increasing amounts of OA from 5 to 

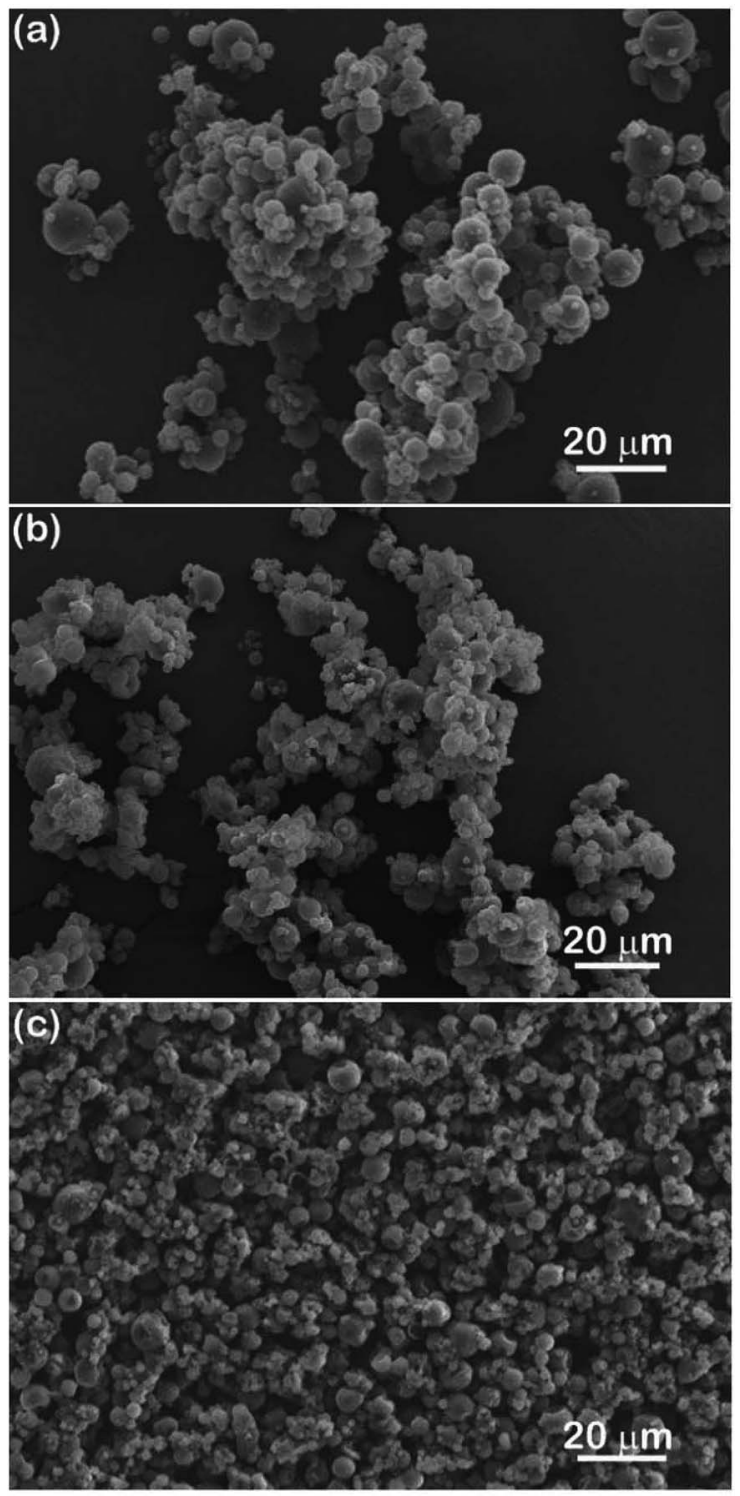

Fig. 4 SEM images showing structural morphology of microcapsules dispersed by 5 wt\% of (a) OA, (b) $\mathrm{OP}_{3}$, and (c) OP.

$20 \mathrm{wt} \%$ indicates that the addition of OA in part provides efficiency to the dispersion of microcapsules in GO, although OA addition was ineffective to the dispersion of microcapsules in toluene in Fig. 3(a).

A gradual decrease in $\eta_{\mathrm{r}}$ value with an increase in dispersant concentration was also observed in the case of $\mathrm{OP}_{3}$ as dispersant, as shown in Fig. 5(b). For the third dispersant, OP, as shown in Fig. 5(c), 5 wt\% addition already caused a significant decrease in $\eta_{\mathrm{r}}$, more so than with $\mathrm{OA}$ and $\mathrm{OP}_{3}$. This result shows that again $\mathrm{OP}$ is more efficient than $\mathrm{OA}$ and $\mathrm{OP}_{3}$ for the dispersion of microcapsules in GO. Although the value of $\eta_{\mathrm{r}}$ continues to decrease with increased amounts of OP, indicating further improved dispersion, further addition is not recommended, since many small organic molecules can act as plasticizers in blended polymers, which can be detrimental to the mechanical properties of coatings.
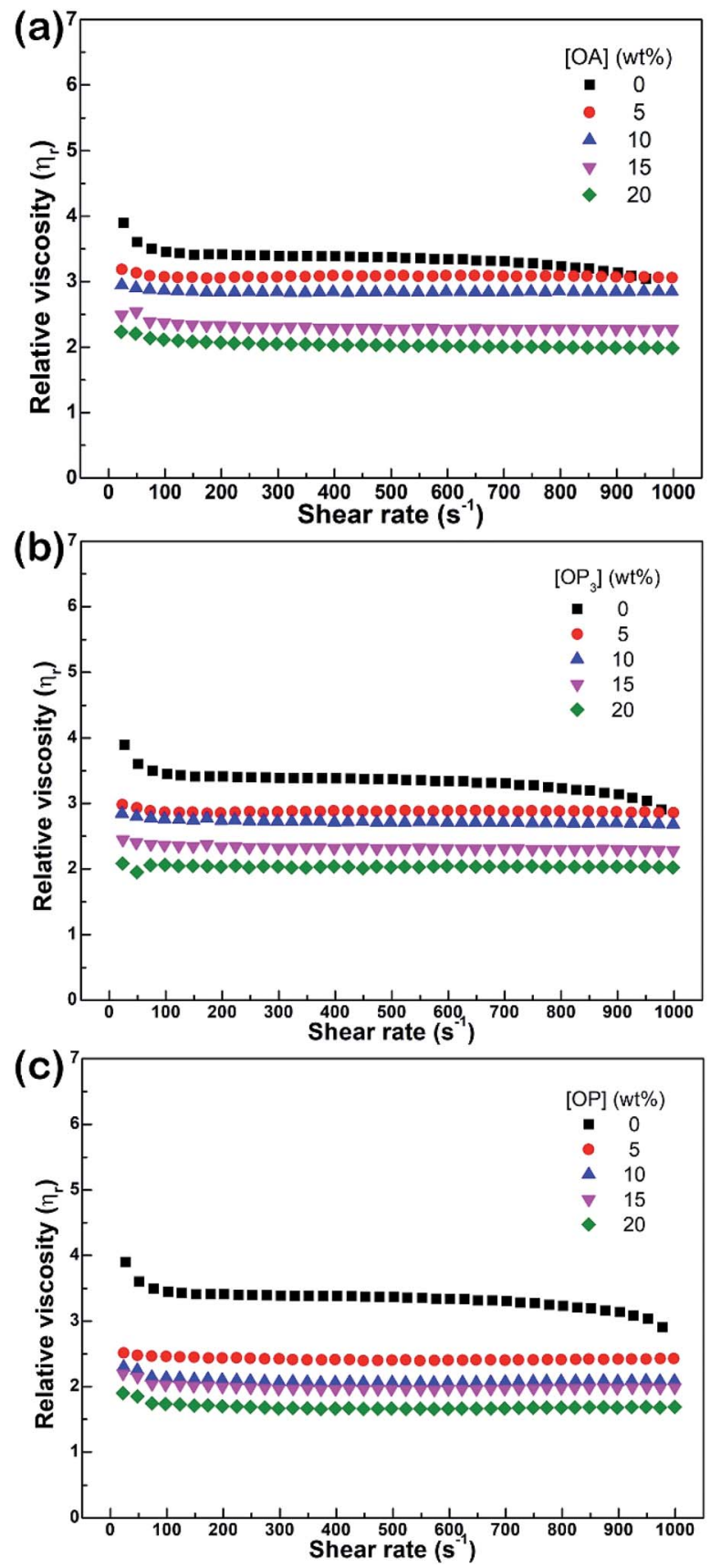

Fig. 5 Relative viscosity as a function of shear rate for a mixture of golden oil and 35 wt\% microcapsules with (a) OA, (b) $\mathrm{OP}_{3}$, and (c) OP dispersants at various concentrations.

\section{Dispersion mechanism}

It is known that particles can be dispersed in a solvent medium through a stabilization mechanism related to electrostatic, steric, electrosteric, or depletion effect. ${ }^{25}$ For the former three mechanisms, attachment/adsorption of the added dispersant on the particle surface is a prerequisite, while this is not required for the depletion dispersion mechanism. For the latter mechanism, the dispersant does not attach to particles but is distributed in the solvent medium. To identify whether the added dispersant is adsorbed onto the microcapsules, Fig. 6(a) 
compares the FT-IR spectra of microcapsules mixed with and without $20 \mathrm{wt} \%$ of different dispersants. For the IR absorption spectrum of the as-received microcapsule in (i), the broad band centered at $3361 \mathrm{~cm}^{-1}$ is attributed to the overlapped $\mathrm{O}-\mathrm{H}$ and $\mathrm{N}-\mathrm{H}$ stretches. ${ }^{27}$ The peaks with strong intensities at 2960, 2916, and $2851 \mathrm{~cm}^{-1}$ correspond to symmetric and asymmetric $\mathrm{C}-\mathrm{H}$ stretches from $-\mathrm{CH}_{2}-$ and $-\mathrm{CH}_{3}$ groups. The IR absorption at $1740 \mathrm{~cm}^{-1}$ is attributed to $-\mathrm{C}=\mathrm{O}$ vibration. The strong peak at $1169 \mathrm{~cm}^{-1}$ is correlated with the $\mathrm{C}-\mathrm{N}$ vibration of the PMF shell. ${ }^{20,27}$ Unexpectedly, all the spectra for microcapsules mixed with dispersants (spectra (ii)-(iv)) show the same IR absorption peaks as in spectrum (i) for the as-received microcapsules, indicating that no adsorption of any of the three dispersants occurs on the microcapsule surface. Since the results of Fig. 3-5 reveal that the addition of OP is effective for the dispersion of the thermochromic microcapsule, the FT-IR spectrum of pure $\mathrm{OP}$ is shown in the spectrum (v) for comparison with spectrum (iv). None of the significant IR peaks corresponding to spectrum (v) can be found in the spectrum (iv), indicating again the absence of OP on the microcapsules. As a result, it can reasonably be concluded that the dispersion of the microcapsules by the addition of OP is dominated by a stabilization mechanism related to the depletion effect.

For depletion stabilization, as illustrated in Fig. 6(b), the non-adsorbed dispersant can generate a repulsion that keeps the microcapsules apart as the microcapsules approach each other. This is because the approach of two microcapsules to each other needs to force out free/non-adsorbed dispersant molecules to create a pure solvent layer between microcapsules, implying de-mixing of the well-dissolved dispersant from the

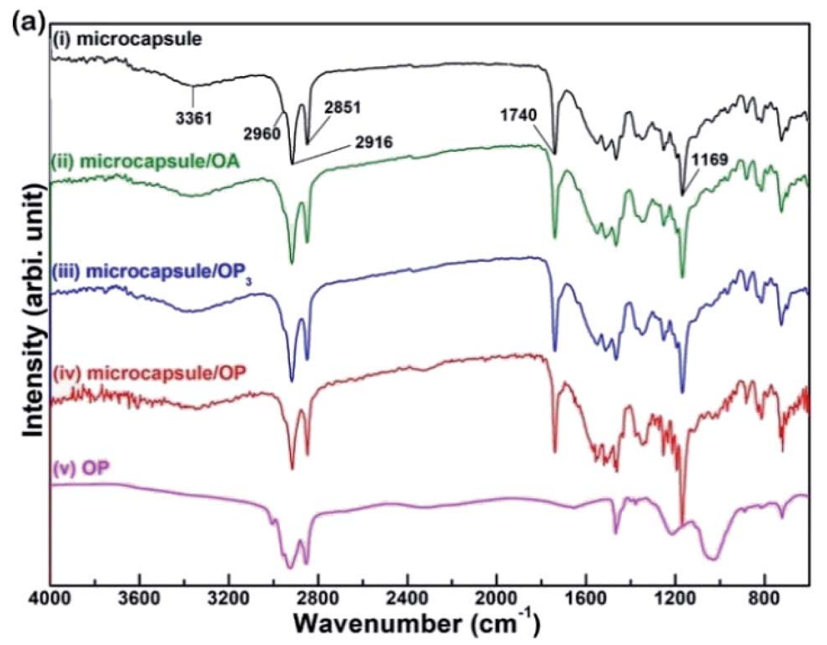

(b)

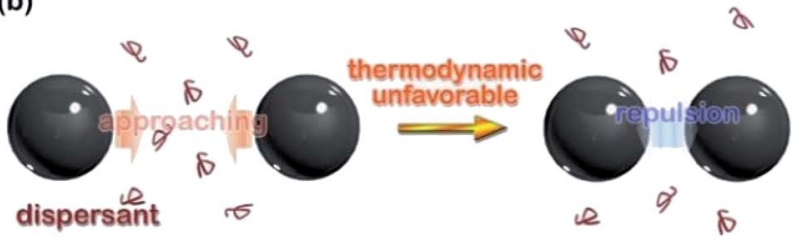

Fig. 6 (a) FT-IR spectra of the (i) as-received, (ii) OA-dispersed, (iii) $\mathrm{OP}_{3}$-dispersed, and (iv) OP-dispersed microcapsules, and (v) pure OP. (b) Schematic showing the depletion dispersion mechanism. solvent, which is a thermodynamically unfavorable process. ${ }^{25} \mathrm{It}$ is believed that dissolved $\mathrm{OA}$ and $\mathrm{OP}_{3}$ are also able to provide depletion force for dispersion of the microcapsules, but their efficiency is poor and distinctive from that of OP, likely attributable to differing solubility of these dispersants in the solvent. According to the literature, ${ }^{28-31}$ the solubility parameters $(\delta)$ of toluene, OA, and OP are 18.2, 15.6, and $17.7(\mathrm{MPa})^{1 / 2}$, respectively. The $\delta$ value of $\mathrm{OP}_{3}$ is not available, but it should be lower than that of OP according to a theoretical calculation based on the group contribution method using eqn (2)-(5): ${ }^{28-30}$

$$
\begin{gathered}
\delta=\delta_{\mathrm{d}}^{2}+\delta_{\mathrm{p}}^{2}+\delta_{\mathrm{h}}^{2} \\
\delta_{\mathrm{d}}=\frac{\sum F_{\mathrm{di}}}{V} \\
\delta_{\mathrm{p}}=\frac{\sqrt{\sum F_{\mathrm{pi}}^{2}}}{V} \\
\delta_{\mathrm{h}}=\sqrt{\frac{\sum E_{\mathrm{hi}}}{V}}
\end{gathered}
$$

where $\delta$ is the complete solubility parameter, and $\delta_{\mathrm{d}}, \delta_{\mathrm{p}}$, and $\delta_{\mathrm{h}}$ are terms correlated to the contributions from dispersive force, permanent dipole-dipole interaction, and hydrogen bonding, respectively. $F_{\mathrm{di}}$ and $F_{\mathrm{pi}}$ are the dispersive and polar terms of the molar attraction constant, and $E_{\mathrm{hi}}$ is the hydrogen bonding term of cohesive energy density; the symbol i represents the terms from different structural groups. Although the parameters listed in Table 1 are insufficient to obtain a complete calculation for both the absolute $\delta$ values of $\mathrm{OP}$ and $\mathrm{OP}_{3}$, a simple estimation based on eqn (2)-(5) and by the limited parameters can still predict that OP would possess a higher value of $\delta$ than $\mathrm{OP}_{3}$. Therefore, of the three dispersants, $\mathrm{OP}$ is the one that exhibits a $\delta$ value closest to that of the solvent medium; i.e., OP has the best solubility in toluene in this investigation, indicating that a higher energy would be required to de-mix $\mathrm{OP}$ than $\mathrm{OA}$ or $\mathrm{OP}_{3}$ from toluene when two microcapsules approach each other. A higher required depletion force is the cause for the superior dispersion efficiency of OP.

\section{Coating and thermochromic performance}

Next, $5 \mathrm{wt} \%$ of different dispersants were added to $35 \mathrm{wt} \%$ microcapsules in GO, cast as films and then crosslinked by UV irradiation for $2 \mathrm{~min}$. Top-surface images of the pure GO film and films of $\mathrm{GO} /$ microcapsules with and without dispersants are shown and compared in Fig. 7(a)-(e). Fig. 7(a) shows that pure GO film is smooth and uniform. When GO is blended with microcapsules, as shown in Fig. 7(b), the surface of the cast film becomes very rough and porous. The roughness would be due to the presence of large agglomerates of microcapsules. The porous nature may be a result of the mixing procedure, because the high content of microcapsules leads to the high viscosity of the $\mathrm{GO} /$ microcapsule mixture and could result in trapping of air during the stirring process. When $\mathrm{OA}$ is used as a dispersant for the mixture, as shown in Fig. 7(c), the cast film exhibits improved surface microstructure, with less roughness and 
Table 1 Parameters for the estimation of $\delta^{30}$

\begin{tabular}{|c|c|c|c|c|}
\hline $\begin{array}{l}\text { Structural } \\
\text { group }\end{array}$ & $F_{\mathrm{di}}\left(J^{1 / 2} \mathrm{~cm}^{3 / 2} \mathrm{~mol}^{-1}\right)$ & $F_{\mathrm{pi}}\left(\mathrm{J}^{1 / 2} \mathrm{~cm}^{3 / 2} \mathrm{~mol}^{-1}\right)$ & $E_{\mathrm{hi}}\left(\mathrm{J} \mathrm{mol}^{-1}\right)$ & $\begin{array}{l}V \\
\left(\mathrm{~cm}^{3} \mathrm{~mol}^{-1}\right)\end{array}$ \\
\hline$-\mathrm{CH}_{3}$ & 420 & 0 & 0 & 33.5 \\
\hline$=\mathrm{CH}-$ & 200 & 0 & 0 & 13.5 \\
\hline$-\mathrm{OH}$ & 210 & 500 & 20000 & 10.0 \\
\hline$-\mathrm{O}-$ & 100 & 400 & 3000 & 3.8 \\
\hline
\end{tabular}

fewer pores. This result agrees well with the slightly improved dispersion that occurs with the addition of $5 \mathrm{wt} \%$ OA in Fig. 5(a). When $\mathrm{OP}_{3}$ is used as the dispersant for the mixture of $\mathrm{GO} /$ microcapsule, the improvements in surface roughness and porosity are similar to what was seen when using OA, as shown in Fig. $7(\mathrm{~d})$. When OP is used as the dispersant (Fig. $7(\mathrm{e})$ ), the film has improved surface flatness, without any pores on the surface, indicating better results than those in the previous two cases with $\mathrm{OA}$ and $\mathrm{OP}_{3}$. This result corresponds well to the superior dispersion efficiency of OP as discussed in the results of Fig. 3-6.

To clarify the dispersion effect of microcapsules on the thermochromic performance of their coatings, the response in color change to variation in temperature for various cast films made of $\mathrm{GO} / \mathrm{microcapsule}$ mixtures with different dispersants was recorded and is compared in Fig. 8. Fig. 8(a) shows the initial state of four $\mathrm{GO} / \mathrm{microcapsule}$ films prior to applying heat. All four films exhibit similar appearance but with differences in surface roughness, as shown in Fig. 7. When a heat source was applied at one end of the samples, as illustrated in Fig. 8(b), placing a block of hot steel $\left(150-200{ }^{\circ} \mathrm{C}\right)$ at one end and a block of cold steel $\left(\sim 25^{\circ} \mathrm{C}\right)$ at the other, the color of the films started to change from black to transparent in the direction of heat flow (Movie S1†). During the heat transfer process, the film with OP-dispersed microcapsules was the first to change color, followed by the films with $\mathrm{OP}_{3}, \mathrm{OA}$, and no dispersant, in that order. Fig. 8(c) shows the intermediate state $40 \mathrm{~s}$ after contact with the hot steel; half of the film has transformed color from black to transparent. Note that the GO used is brown in color and hence renders the films slightly brown after the color change to transparent upon heating. Interestingly, for the film fabricated with OP-dispersed microcapsules, the color transformed in a more uniform way with a horizontal front, revealing the letters "OP" behind the film more quickly. For the films containing non-dispersed and OA-dispersed microcapsules, the color changed non-uniformly and at a much slower pace.

After contact with heat for $100 \mathrm{~s}$, as shown in Fig. 8(d), all specimens, except that containing non-dispersed microcapsules, show the completed process of color transformation; the film with OP ended after $\sim 70 \mathrm{~s}$, the film with $\mathrm{OP}_{3}$ ended after $\sim 80 \mathrm{~s}$, and the film with OA ended after $\sim 90 \mathrm{~s}$. It can also be seen that the specimen containing OP-dispersed microcapsules exhibits the best brightness and transparency after the color

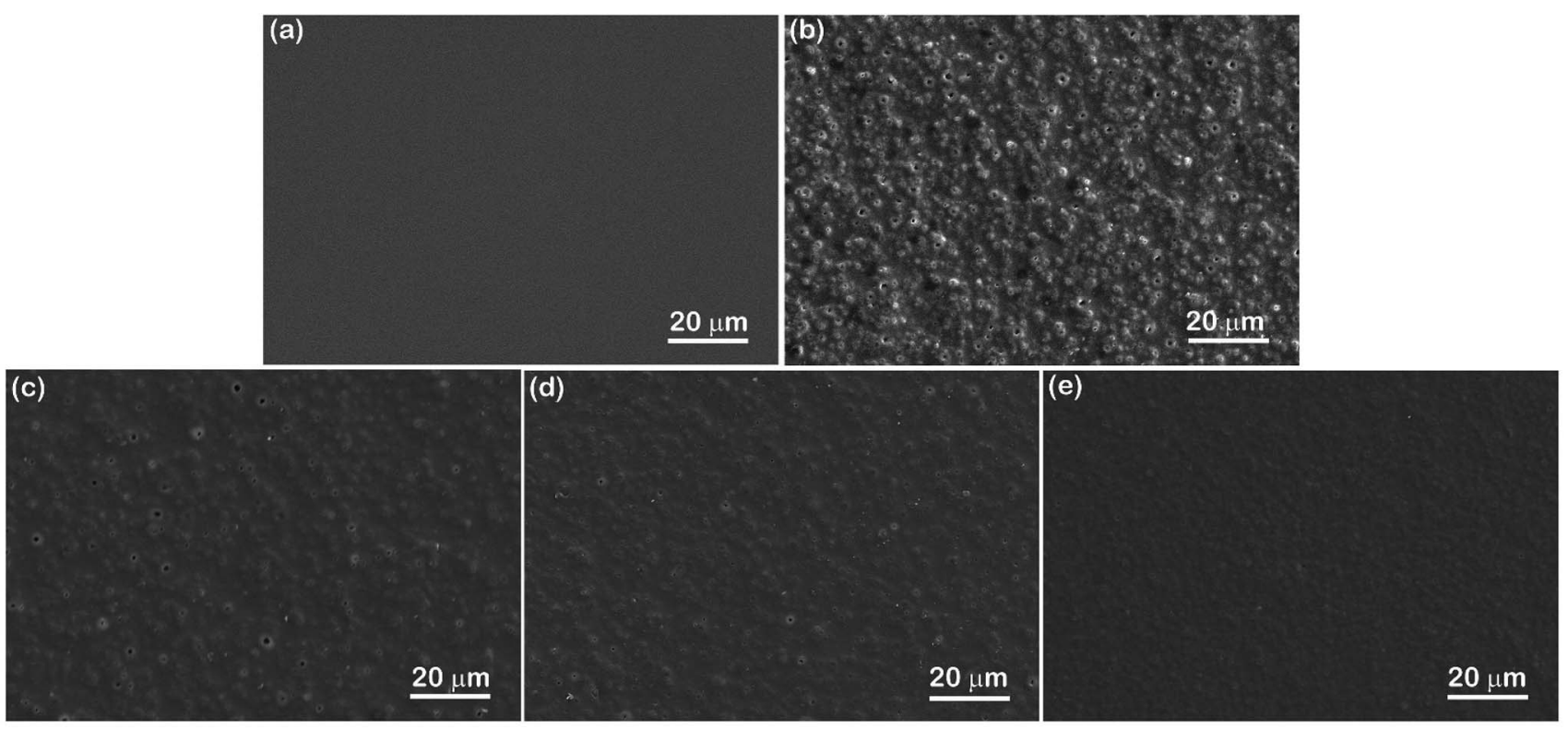

Fig. 7 SEM images showing the surface microstructure of cast films of (a) golden oil, and golden oil mixed with 35 wt\% microcapsules with (b) no dispersant or $5 \mathrm{wt} \%$ of (c) OA, (d) $\mathrm{OP}_{3}$, and (e) OP. 
(a)

transformation, while the specimens containing non- and OAdispersed microcapsules are darker and less transparent, rendering the letters "none" and "OA" printed behind them somewhat obscure. For the specimens containing non- and OAdispersed microcapsules, the slower and non-uniform color change can be attributed to poorer dispersion of the microcapsules. This is because the speed of heat transfer would be different when comparing microcapsules inside an agglomerate to those between agglomerates. Also, heavy agglomeration of microcapsules would leave part of the film deficient in microcapsule content, which would result in slower response and non-uniform change in color. The low transparency found in the two films with non- and OA-dispersed microcapsules can be correlated to the presence of agglomerates and pores in the film microstructure, since both would cause more light scattering in the film, leading to less transparency. In summary, all the results herein clearly demonstrate the significance of microcapsule dispersion on coating quality and thermochromic performance.

\section{Experimental}

\section{Raw materials}

A commercially available thermochromic microcapsule (TMPDF 31, New Prismatic Enterprise Co., Ltd., Taiwan) with a particle size range of $2-10 \mu \mathrm{m}$ was used. Three surfactants including oleic acid (OA) (70\%; Sigma-Aldrich Co., Steinheim, Italy), oleyl phosphate (OP) (85\%; TCI, Tokyo, Japan), and tris(2-butoxyethyl) phosphate $\left(\mathrm{OP}_{3}\right)$ (95\%; TCI, Tokyo, Japan) were showing color transforming from black to transparent in the direction of heat flow. (b)

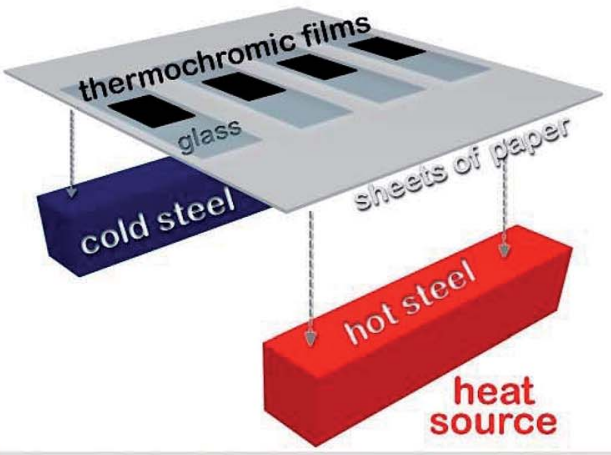

(d)

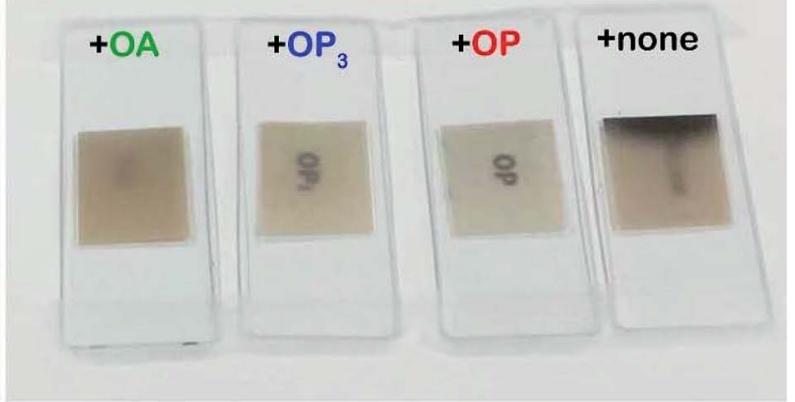

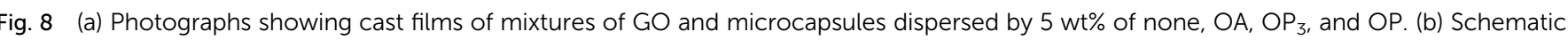

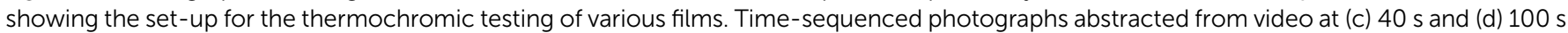

used as dispersants. A UV-curable golden oil (GO) (F24, New Prismatic Enterprise Co., Ltd., Taiwan) was used as the dispersion medium. Toluene (99\%, ECHO, Toufen, Taiwan) was used as a dilution solvent for the dispersion medium.

\section{Characterizations}

To investigate the dispersion stability of the thermochromic microcapsule in toluene, a sedimentation experiment was conducted on the microcapsule/toluene suspension for different time periods. Note that the concentration of the microcapsule is $35 \mathrm{wt} \%$ based upon the weight of dispersion medium and the added dispersants are $0-20 \mathrm{wt} \%$ based upon the weight of microcapsules, and all concentrations of microcapsules and dispersants mentioned in this paper are calculated on the same basis. To characterize the dispersion of microcapsules in GO, mixtures containing GO and $35 \mathrm{wt} \%$ microcapsules were prepared and their rheology was measured using a concentric cylinder rheometer (AR1000, TA Instruments Ltd., UK). The interaction between the different dispersants and the microcapsules was characterized by Fourier transform infrared spectroscopy (FT-IR; DA 8.3, Bomen, Canada); $20 \mathrm{wt} \%$ of each dispersant was mixed with $35 \mathrm{wt} \%$ microcapsules in toluene for one week, and then the microcapsules were separated from the suspension for FT-IR characterization. To prepare thermochromic coatings, GO was mixed with $35 \mathrm{wt} \%$ microcapsules and $5 \mathrm{wt} \%$ dispersant and then the mixture was stirred gently for $10 \mathrm{~min}$. The resulting mixture was cast on a glass slide and solidified by UV irradiation $\left(88 \mathrm{~W} \mathrm{~cm}^{-2}\right)$ for $2 \mathrm{~min}$; the resulting film had an average thickness of $60 \mu \mathrm{m}$. The 
morphology of the microcapsules and the microstructure of the cast films were examined using a field-emission scanning electron microscope (SEM; S-470, Hitachi, Tokyo, Japan). To measure thermochromic performance, one end of the various cast films was contacted with hot steel to record the color transformation.

\section{Conclusions}

The efficiency of three dispersants, $\mathrm{OA}, \mathrm{OP}_{3}$, and $\mathrm{OP}$ for improving dispersion of a commercially available thermochromic microcapsule consisting of a PMF-based shell was studied and compared, and the importance of the dispersion of these microcapsules for its application in the thermochromic coating was determined in this investigation. According to sedimentation and SEM imaging results, it was found that the microcapsules naturally agglomerate, and application of high-power ultrasonication in dilute solvent is not helpful for their deagglomeration. Among the three investigated dispersants, the addition of OP was the most effective for the dispersion of the microcapsule in both toluene and golden oil for thermochromic coatings. From the results of rheological and FT-IR characterizations, it was found that none of the three dispersants adsorbed onto the microcapsule surface; therefore, it is proposed that the most reasonable mechanism for good dispersion of the microcapsule by the use of OP is the depletion stabilization effect. Based on this dispersion mechanism, the approach of microcapsules can be prevented through a repulsion effect to prevent agglomeration, due to the good solubility of $\mathrm{OP}$ in the solvent medium. $\mathrm{OA}$ and $\mathrm{OP}_{3}$ are less soluble in the dispersion solvent used when compared to OP, and hence exhibit less efficiency for microcapsule dispersion. With improved dispersion of microcapsules, cast films of $\mathrm{GO}$ / microcapsules showed not only better surface flatness and less porosity but also the excellent thermochromic performance with faster and more uniform color change, resulting in higher transparency and brightness of the color-transformed film.

\section{Conflicts of interest}

There are no conflicts to declare.

\section{Acknowledgements}

The authors are grateful for the financial support from the National Taipei University of Technology.

\section{References}

1 C. M. Dry and N. R. Sottos, Passive smart self-repair in polymer matrix composite materials, in North American Conference on Smart Structures and Materials 1993, Albuquerque, NM, United States, 1993, vol. 1916, pp. 438445.

2 S. R. White, N. R. Sottos, P. H. Geubelle, J. S. Moore, M. Kessler, S. R. Sriram, E. N. Brown and S. Viswanathan, Nature, 2001, 409, 794.
3 S. van Der Zwaag and E. Brinkman, Self Healing Materials: Pioneering Research in The Netherlands, IOS Press, The Netherlands, 2015.

4 S. K. Ghosh, Self-Healing Materials: Fundamentals, Design Strategies, and Applications, John Wiley \& Sons, New York, 2009.

5 B. J. Blaiszik, S. L. Kramer, M. E. Grady, D. A. McIlroy, J. S. Moore, N. R. Sottos and S. R. White, Adv. Mater., 2012, 24, 398.

6 Y. J. Lan, S. J. Chang and C. C. Li, J. Mater. Chem. A, 2017, 5, 25583.

7 S. H. Cho, S. R. White and P. V. Braun, Adv. Mater., 2009, 21, 645.

8 H. Wei, Y. Wang, J. Guo, N. Z. Shen, D. Jiang, X. Zhang, X. Yan, J. Zhu, Q. Wang, L. Shao, H. Lin, S. Wei and Z. Guo, J. Mater. Chem. A, 2015, 3, 469.

9 F. Zhang, P. Ju, M. Pan, D. Zhang, Y. Huang, G. Li and X. Li, Corros. Sci., 2018, 144, 74.

10 M. Odarczenko, D. Thakare, W. Li, K. Yang, S. Tang, S. P. Venkateswaran, N. R. Sottos and S. R. White, ACS Omega, 2018, 3, 14157.

11 T. Nesterova, K. Dam-Johansen, L. T. Pedersen and S. Kiil, Prog. Org. Coat., 2012, 75, 309.

12 C. A. Chen and C. C. Li, Solid State Ionics, 2018, 323, 56.

13 P. H. Huang, S. J. Chang and C. C. Li, J. Power Sources, 2017, 338, 82.

14 M. N. Singh, K. S. Y. Hemant, M. Ram and H. G. Shivakumar, Res. Pharm. Sci., 2010, 5, 65.

15 C. A. Chen, C. C. Li and C. H. Chen, Mater. Horiz., 2018, 5, 1092.

16 S. Sergeeva, D. A. Gorin and D. V. Volodkin, Bionanoscience, 2014, 4, 1.

17 E. Koh, N. K. Kim, J. Shin and Y. W. Kim, RSC Adv., 2014, 4, 16214.

18 F. Alizadegan, S. M. Mirabedini, S. Pazokifard, S. G. Moghadam and R. Farnood, Prog. Org. Coat., 2018, 123, 350.

19 J. Hu, X. Zhang, J. Qu, Y. Wen and W. Sun, Ind. Eng. Chem. Res., 2018, 57, 3591.

20 M. Vukoje, S. Miljanić, J. Hrenović and M. Rožić, Cellulose, 2018, 25, 6121.

21 H. Li, R. Wang, H. Hu and W. Liu, Appl. Surf. Sci., 2008, 255, 1894.

22 X. M. Tong, M. Zhang, M. S. Wang and Y. Fu, J. Appl. Polym. Sci., 2013, 127, 3954.

23 J. Li, M. J. Mazumder, H. D. Stöver, A. P. Hitchcock and I. M. Shirley, J. Polym. Sci., Part A: Polym. Chem., 2011, 49, 3038.

24 D. J. Shaw and B. Costello, Introduction to Colloid and Surface Chemistry, Butterworth-Heinemann, Oxford, 1991.

25 E. Kissa, Dispersions, Characterization, Testing, and Measurement, Marcel Dekker Inc., New York City, 1999.

26 T. Sato and R. Ruch, Stabilization of Colloidal Dispersions by Polymer Adsorption, Marcel Dekker, NY, 1980.

27 G. Socrates, Infrared and Raman Characteristic Group Frequencies-Tables and Charts, John Wiley \& Sons, NY, 3rd edn, 2001. 
28 C. M. Hansen, Hansen Solubility Parameters: A User's Handbook, CRC press, 2002.

29 B. A. Miller-Chou and J. L. Koenig, Prog. Polym. Sci., 2003, 28, 1223.
30 E. A. Grulke, Solubility parameter values, in, Polymer Handbook, ed. J. Brandrup, and E. H. Immergut, Wiley, New York, 3rd edn, 1989, pp. 519-557.

31 M. S. Kuo, S. J. Chang, P. H. Hsieh, Y. C. Huang and C. C. Li, J. Am. Ceram. Soc., 2016, 99, 445. 